\title{
Resistance to sharp eyespot in wheat
}

\author{
M.G. Cromey, C.C.L. Hide and E.D. Meenken \\ The New Zealand Institute for Plant E Food Research Limited, Private Bag 4704, \\ Christchurch, New Zealand \\ Corresponding author: matthew.cromey@plantandfood.co.nz
}

\begin{abstract}
Susceptibility of 16 wheat cultivars to sharp eyespot (caused by Rhizoctonia cerealis) was examined in field experiments. Disease incidence varied markedly between experiments, but one cultivar, 'Regency', consistently had very low incidence, which was confirmed in a greenhouse experiment. Some cultivars had a high incidence of disease in some field experiments, but most cultivars had intermediate incidence. A greenhouse experiment provided some evidence for pathogenic variation in $R$. cerealis. Monitoring of disease progression in commercial crops confirmed the cultivar differences identified in field experiments. Infection was greater in crops sown in mid-autumn than in late-autumn to winter. Primary infection occurred during seedling growth stages, with maximum disease incidence occurring by late spring. Primary infection developed in resistant cultivars, but there was little spread to inner leaf sheaths. Selection for resistance to sharp eyespot is feasible in breeding programmes and commercial losses could be reduced by using the more resistant cultivars.
\end{abstract}

Keywords Rhizoctonia cerealis, disease progress, cultivars, resistance.

\section{INTRODUCTION}

Sharp eyespot, caused by Rhizoctonia cerealis van der Hoeven, is common in wheat (Triticum aestivum) crops in many wheat-growing regions of the world, including New Zealand (Cromey et al. 2002), the United Kingdom (Clarkson \& Cook 1983) and China (Shi et al. 2000). The disease was recorded in $43 \%$ of surveyed New Zealand wheat crops in the 1998/99 growing season, and its incidence in infected crops ranged from 2 to $88 \%$ over the period 1996-2000 (Cromey et al. 2002). Lesions usually begin on the outer leaf sheaths near the base of the plant and can progressively extend through the leaf sheath layers, infecting the stem and pith in severe cases (Pitt 1964). Severe sharp eyespot infection of mature tillers may result in small, shrivelled grain, lodging and premature ripening (Clarkson \& Cook 1983). While sharp eyespot is not a major yield-limiting disease of wheat, losses in individual crops in New Zealand have been calculated to be as high as $18 \%$ (Cromey et al. 2002).

There is little information on cultivar resistance to sharp eyespot (Wiese 1987). The use of resistant cultivars, or at least the avoidance of highly susceptible cultivars, may be a useful strategy to minimise the risk of yield losses from this disease. Differences in susceptibility were found between cultivars in field trials carried out in the late 1970s and early 1980s in England (Hollins \& Scott 1983; Clulow \& Wale 1984). While some cultivars were always relatively heavily or lightly infected, the ranking order 
of cultivars was not always consistent. More recently, cultivar resistance in wheat to sharp eyespot has been examined in China. Differences in resistance between cultivars were identified, with cultivars ranging from highly resistant to highly susceptible, although only $4-7 \%$ of all wheat lines were tested, and as few as $1.7 \%$ of commercial cultivars were resistant ( $\mathrm{He}$ et al. 1998; Shi et al. 2000).

An initial study suggested that New Zealand wheat cultivars differed in resistance to sharp eyespot (Cromey et al. 2005). In the current study, the resistance of wheat cultivars grown in New Zealand to sharp eyespot was investigated in field and greenhouse experiments.

\section{MATERIALS AND METHODS Cultivar field trials}

Assessments of sharp eyespot in 15 wheat cultivars were carried out in six field trials in Canterbury, New Zealand, in the 2005/06 growing season. Details of cultivar and trial location are show in Figure 1. The trials were part of a series of cultivar evaluation trials located within commercial wheat fields on New Zealand growers' properties. Trials were sown in autumn (April or May) in randomised complete block designs with four replicates, and consisted of nine rows, $8 \mathrm{~m}$ long. Sharp eyespot incidence in each plot was assessed in situ at the soft dough stage of grain development (GS 85, Zadoks et al. 1974). Incidence (the percentage of tillers with distinct sharp eyespot lesions on outer leaf sheaths) was assessed by counting tillers (infected and healthy) in 0.5-m lengths of row (avoiding the outer two rows) in each of four positions in each plot.

\section{Lincoln cultivar trials}

Two adjacent trials were sown at the Plant \& Food Research (PFR) research farm at Lincoln, Canterbury, in 2005. The first trial was sown on 4 April (mid-autumn) and the second on 27 May (late-autumn). Each trial consisted of 12 wheat cultivars sown in complete block designs with four replicates. Each plot had three rows, $1 \mathrm{~m}$ long. Plots were harvested at ground level at GS 85 (January 2006). Each tiller was assessed for the presence of distinct sharp eyespot lesions on the leaf sheath, and then leaf sheaths were removed and the plants assessed for the presence of sharp eyespot lesions on the stem. Results were recorded as the incidence of disease ( $\%$ tillers with lesions) and incidence on stems (\% stems with lesions).

\section{Disease progress in commercial wheat crops}

Sharp eyespot disease progress was monitored in 40 commercial Canterbury wheat crops in the 2005/06 growing season. Four crops were of the resistant cultivars 'Regency' or 'Torlesse' (both had consistently low disease severity in field trials), while the remaining 36 crops were of more susceptible cultivars, with 'Macro' and 'Savannah' being the most commonly planted. Samples were collected monthly (five in total) between September (stem elongation stage of crop growth) and January (mid dough stage) from each crop. Samples were taken along a W sampling pattern from a designated 0.6-ha area of each crop, the site being recorded using GPS. Samples of five plants were collected from each of 10 positions along the $\mathrm{W}$. Plants were taken to the laboratory where each tiller was assessed for the presence of sharp eyespot lesions on each of the outer four leaf sheaths.

\section{Greenhouse experiment 1}

The effects of different rates of $R$. cerealis inoculum on two wheat cultivars were examined in a greenhouse experiment. The objective of this experiment was to determine whether the differences in disease expression between these cultivars observed in field trials could be repeated in the greenhouse, and to determine the optimum inoculum loading to discriminate between cultivars in terms of resistance.

The trial had two cultivars ('Regency', which had low disease incidence in field trial and 'Equinox', which had higher incidence) with four rates of inoculum (0, 20, 50 and $80 \mathrm{~g} / 8$-litre pot), giving a total of 8 treatments. Inoculum was prepared by growing an isolate of $R$. cerealis (from a sharp eyespot lesion on 'Equinox' at Lincoln, Canterbury) in a 20:1 mixture of sieved sand and cornmeal (autoclaved for 
35 min at $121^{\circ} \mathrm{C}$ ) for 2 weeks. Pots were filled with potting mix. Inoculum was applied to pots immediately before planting by sprinkling the appropriate quantity on the surface and lightly mixing into the surface to a depth of $2 \mathrm{~cm}$. Seeds were germinated on filter paper and vernalised for 8 weeks at $4^{\circ} \mathrm{C}$ before being transferred to prepared pots. Control pots were included in the trial to ensure that the inoculum treatment was the true source of infection in treated pots, but were excluded from the final statistical analysis. There were four replicates of each treatment in a completely randomised design. The greenhouse was maintained at a constant temperature of approximately $18^{\circ} \mathrm{C}$ with $12 \mathrm{~h}$ light and $12 \mathrm{~h}$ dark. Each pot contained three plants, some of which died in some pots (plant death was not due to sharp eyespot). At the soft dough stage of grain development, each tiller was assessed for the percent area covered with sharp eyespot lesions. Each pot contained between 10 and 20 tillers. Analysis was carried out on the average percent stem area affected per pot.

\section{Greenhouse experiment 2}

To investigate the possibility of pathogenic variation, the effects of different isolates of $R$. cerealis on five wheat cultivars were assessed in a greenhouse experiment. The isolates were from sharp eyespot lesions in wheat cultivar trials at Lincoln in central Canterbury (from 'Equinox'), Chertsey (from 'Alberic') and Methven (from 'Macro') in mid-Canterbury, and St Andrews (from 'Savannah') in South Canterbury. These isolates were chosen because they were from cultivars that were susceptible at the particular location. The cultivars tested were 'Regency', 'Alberic', 'Equinox', 'Savannah' and 'Phoenix'. The objective was to determine if the cultivars differed in susceptibility to sharp eyespot, whether the isolates differed in aggressiveness and whether there was evidence for cultivar $x$ isolate interactions. Inoculum preparation, planting and assessments were carried out as in Greenhouse Experiment 1. A single rate of inoculum of $80 \mathrm{~g} /$ pot was used for of each of four isolates, each from a different geographic
location).There were a few missing values where all plants in a pot were dead. Assessments were the same as for experiment 1 .

\section{Statistical methods}

The incidence of sharp eyespot in the field trials and commercial crop samples was analysed using a Generalised Linear Mixed Model (Schall 1991) for binomially distributed data with a logit link. This included replicates as random effects and treatments or cultivars as fixed effects. For the greenhouse experiments, the data were analysed using a Generalised Linear Model with a logit link (McCullagh \& Nelder 1989). Comparisons between cultivars or inoculum concentration were assessed with F-tests within the analyses of deviance done as part of the analyses. All analyses used GenStat v.10 or v.11. Results are presented on the natural scale with $95 \%$ confidence intervals for maximum and minimum predicted values within a graph. For the cultivar trials, cultivars with zero infection in all replicates were removed from the data before formal statistical analysis. Similarly for the inoculated field trial, treatment combinations with zero means were removed before analysis.

\section{RESULTS}

\section{Cultivar trials}

The mean incidences (as \% tillers infected) and associated confidence intervals of sharp eyespot in 15 wheat cultivars differed markedly between the six field trials (Figure 1). The highest disease incidences were observed in trial A3, where one cultivar ('Regency') had less than 10\% infected tillers, but six of the 16 cultivars had at least $50 \%$ tillers infected. There was strong evidence that the incidence of sharp eyespot differed between at least some of the cultivars $(\mathrm{P}<0.001)$. Disease incidence was low in 'Regency' in all trials, and also consistently low in 'Option'. 'Phoenix' was the most heavily infected cultivar in all six trials, with incidence ranging from $19 \%$ to $84 \%$ of tillers infected.

Most cultivars had low to moderate sharp eyespot incidence in most trials, but some cultivars had relatively high incidence in one 
or more trials. For instance, 'Savannah' (in trial A1), 'Claire' (trial A5) and 'Alberic' (A3) were each amongst the most heavily infected in their respective trials, while 'Tanker' had relatively high disease incidence in two trials (A1 and A3).The most heavily infected trial was located at Methven, Canterbury (Trial A3) and was April-sown. The two least infected trials were the two trials sown in May, one in Wakanui, mid Canterbury (Trial A2), and the other in St Andrews, South Canterbury (Trial A4).

\section{Lincoln cultivar trials}

Disease incidence was much greater in the midautumn April-sown trial at Lincoln than in the late-autumn May-sown trial, in particular in the incidence of tillers with lesions through to the stem (Figure 2). The highly susceptible cultivar Phoenix was not available at the time of this trial. In April-sown plots, cultivar ranking based on the incidence of lesions on stems was

A2

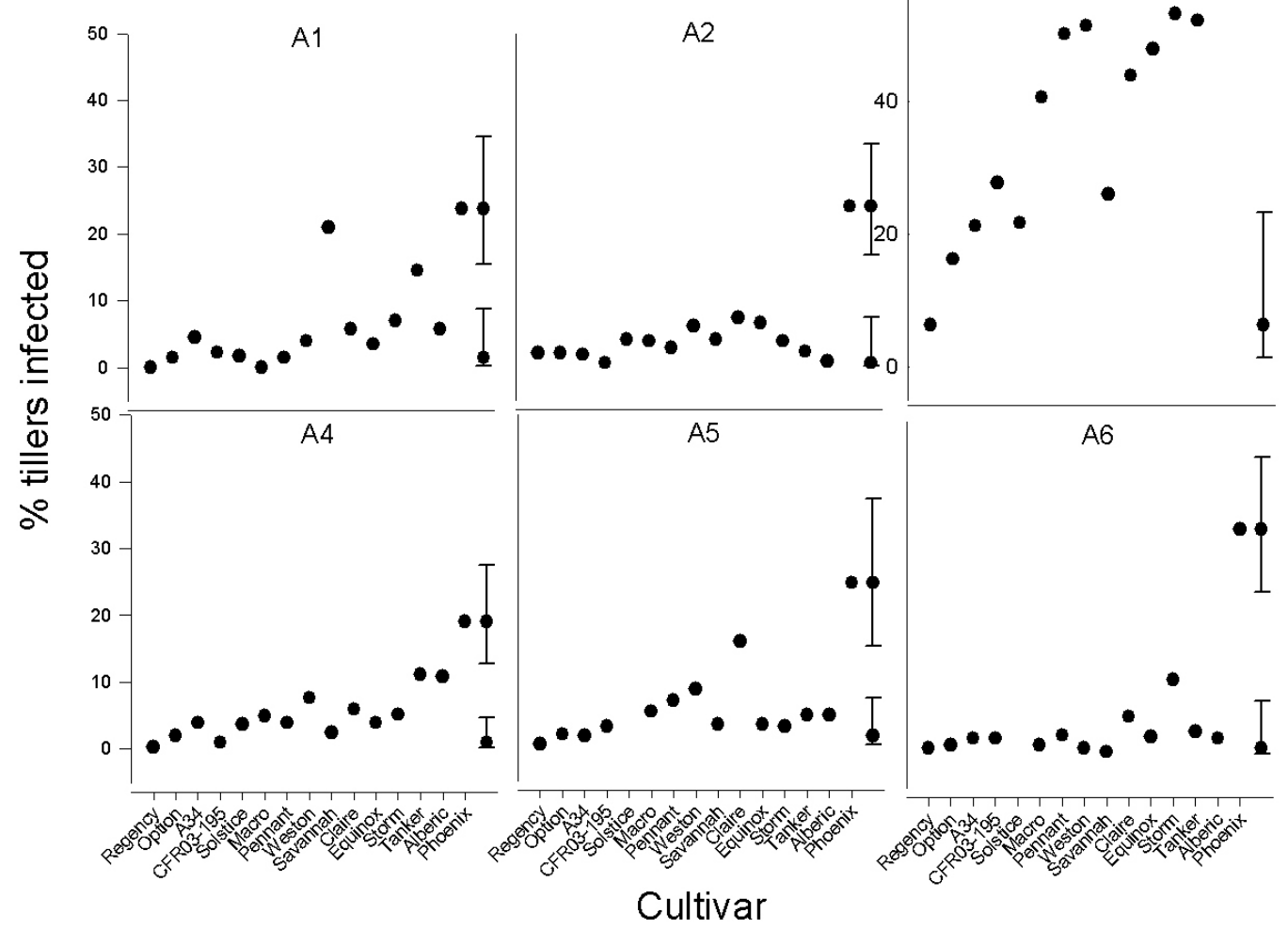

Figure 1 Mean incidence of sharp eyespot (\% tillers infected) in 15 wheat cultivars in six field trials sown in 2005. Location and sowing dates were: A1, Chertsey (mid Canterbury), 5 April; A2, Wakanui (mid Canterbury), 6 May; A3, Methven (mid Canterbury), 27 April; A4, St Andrews (South Canterbury), 7 May; A5, Balfour (Southland), 20 April; A6, Oreti (Southland), 20 April. Bars represent 95\% confidence intervals for the maximum and minimum predicted values. 
different from that based on leaf sheath lesions. For instance, 'Macro' had the second highest proportion of tillers with lesions on the leaf sheath, but had a relatively low proportion of lesions reaching the stem. In May-sown plots, although some cultivars had over $10 \%$ of tillers infected, very few lesions extended through to the stem. While the incidence on stems ranged between $1 \%$ and $14 \%$ for cultivars in the Aprilsown trial, variability between plots resulted in large confidence intervals.

\section{Disease progress in commercial wheat crops}

Changes in sharp eyespot incidence were monitored in March-sown (5 crops), April-sown (12 crops), May-sown (13 crops) and June-sown (6 crops) crops of susceptible cultivars (Figure 3). Data were examined separately for the most common cultivars 'Macro' and 'Savannah', and showed the same pattern across months (data not presented) as the full cultivar set, so the complete data set was used for analysis. The incidence was highest in April-sown crops. For example, mean incidence at the first assessment date (September) was $46 \%$ in April-sown crops, compared with 11\%, 12\% and 6\% for March-, May- and June-sown crops respectively.

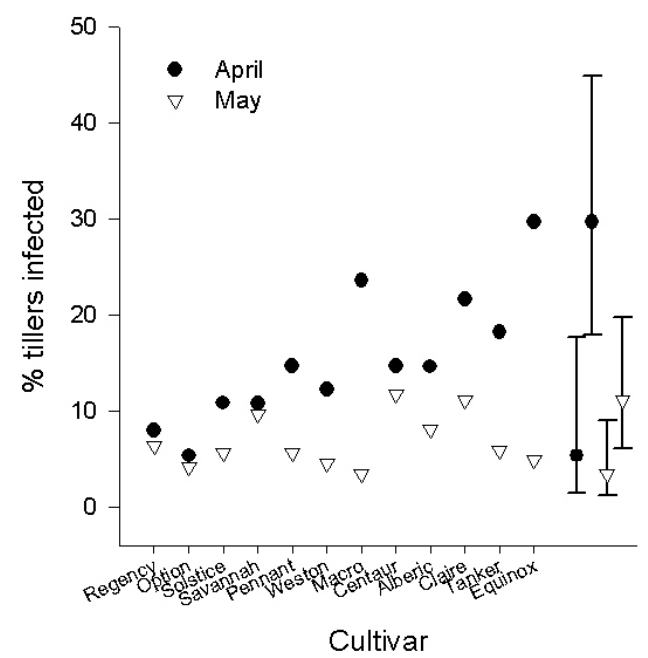

The proportion of tillers infected in Aprilsown crops of susceptible cultivars was similar at the first three assessment dates. At the first two assessments, most infection was restricted to the outer leaf sheath. By November, the proportions of tillers with lesions on inner leaf sheaths increased, and by January most tillers with visible lesions had lesions through to at least the fourth sheath.

In March and May-sown crops, sharp eyespot incidence in crops of susceptible cultivars increased steadily until the November assessment. The incidence of lesions on inner leaf sheaths did not increase substantially until the January assessment, during the later stages of grain fill. Changes in sharp eyespot incidence in June-sown crops followed a similar pattern to that of May-sown crops, although incidence peaked at the October sampling, rather than the November sampling.

Sharp eyespot incidence was less in December than in earlier assessments for all sowing dates. This probably coincides with the death of outer leaves and leaf sheaths, and loss of visible disease on the dead sheaths. Incidence then increased again during grain fill and most lesions extended through at least four leaf sheaths.

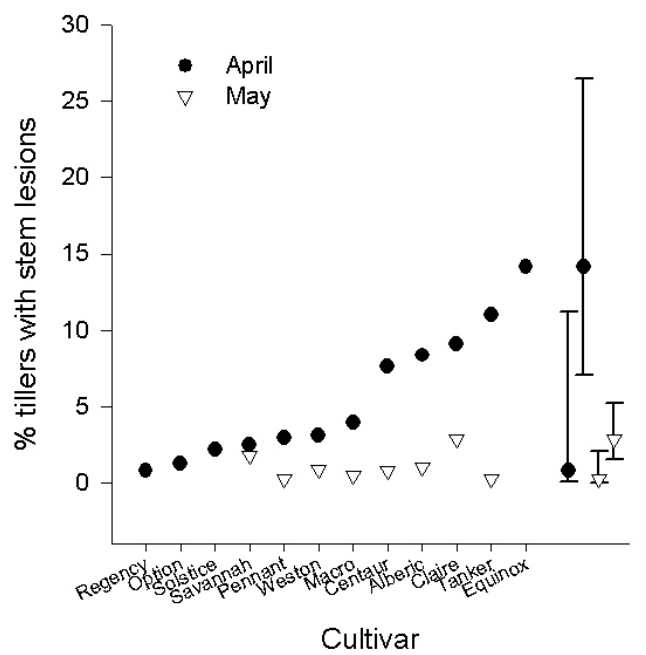

Figure 2 Sharp eyespot incidence (\% tillers infected and \% tillers with stem lesions) in 12 wheat cultivars in April or May 2005 sown trials at Lincoln, Canterbury. Bars represent 95\% confidence intervals for the maximum and minimum predicted values within each sowing date. 
Susceptible cultivars: March sown

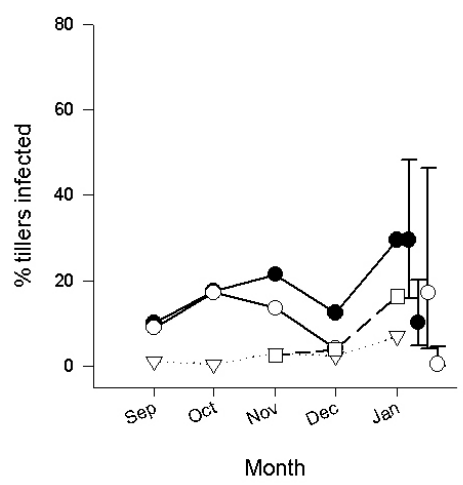

Susceptible cultivars: June sown

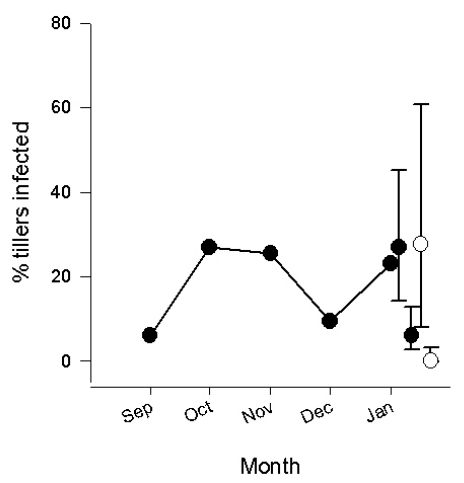

Susceptible cultivars: April sown

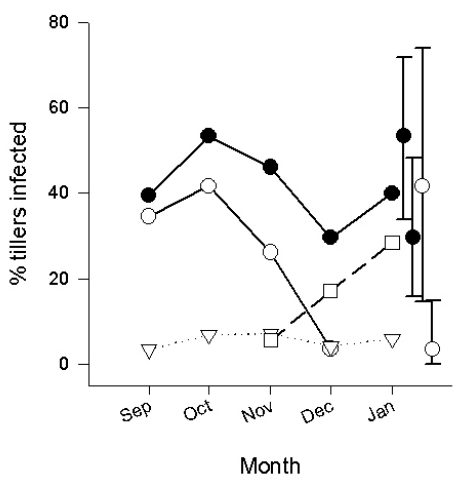

Resistant cultivars: May sown

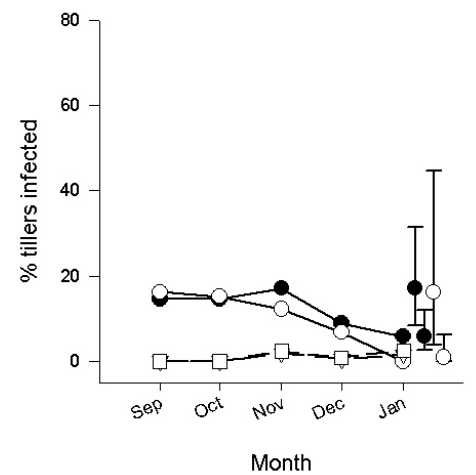

Susceptible cultivars: May sown

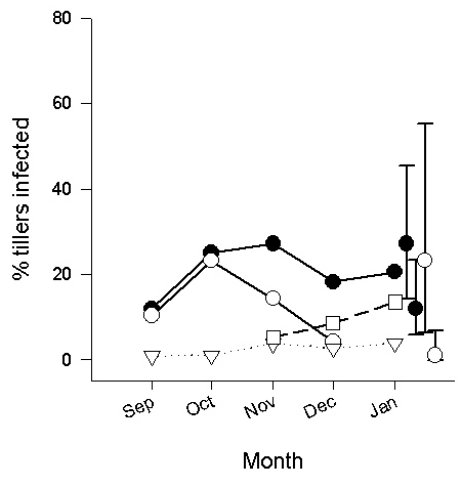

Resistant cultivars: June sown

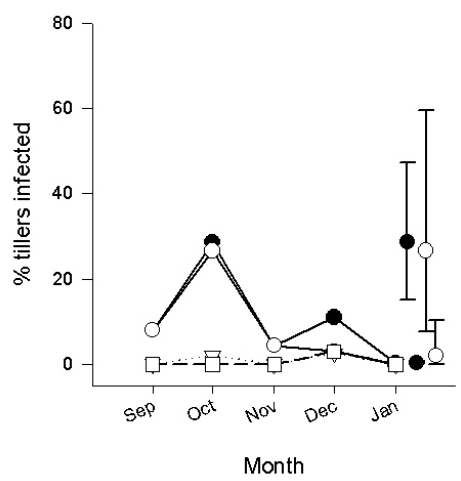

Figure 3 Sharp eyespot disease progress in crops of susceptible wheat cultivars sown in March, April, May or June 2005 or resistant cultivars sown in May or June 2005. Bars represent 95\% confidence intervals for the maximum and minimum predicted values.

Sharp eyespot incidence was examined in four crops of cultivars that had consistently low disease incidence in cultivar trials (Figure 3). Three crops were (autumn) May-sown (two 'Regency' and one 'Torlesse'), while one ('Torlesse') was (winter) June-sown. Disease incidence was similar to that of susceptible cultivars during the first two assessment dates but was less in following assessments, with infection remaining largely restricted to outermost leaf sheaths, which senesced and were lost. In Maysown crops, disease incidence dropped after the November assessment, probably as outer leaves died and infection did not move into younger leaf sheaths. In the single June-sown crop, incidence peaked at $29 \%$ in the October assessment and then dropped to low levels in subsequent assessments. By the final assessment in January, no disease was recorded.

\section{Greenhouse experiment 1}

The percentage area of outer leaf sheaths infected with sharp eyespot in the first greenhouse experiment was higher for 'Equinox' than for 'Regency' ( $\mathrm{P}<0.001)$. The mean area affected 
was $28 \%$ and $7 \%$ for 'Equinox' and 'Regency' respectively. Percentage area infected varied little between the rates of inoculum ( $P=0.642)$, with a similar pattern for both cultivars $(\mathrm{P}=0.593$ for the cultivar by inoculum interaction).

\section{Greenhouse experiment 2}

Sharp eyespot severity (percent stem area affected) varied significantly between cultivars $(\mathrm{P}<0.001$, Figure 4), with 'Phoenix' and 'Savannah' most severely infected. Sharp eyespot severity on 'Alberic' was highest with the Chertsey isolate (isolated from 'Alberic'), and on 'Equinox' was highest with the Lincoln isolate (isolated from 'Equinox'). There was also an indication ( $\mathrm{P}$ $=0.088$ ) that the average percent stem area affected also differed between isolates, with the Chertsey isolate resulting in a higher percent area affected than the other isolates on four of the five cultivars.

\section{DISCUSSION}

Results of field trials, greenhouse experiments and comparisons of cultivars in commercial crops have demonstrated that wheat cultivars grown in New Zealand differ in their resistance to sharp eyespot. One cultivar ('Regency') in particular had consistently low sharp eyespot incidence in field trials, confirming the results of an initial study (Cromey et al. 2005). Other cultivars often had relatively high incidence. However, most cultivars were intermediate in their susceptibility to the disease. A greenhouse experiment provided further evidence for the resistance of 'Regency' under controlled conditions. Inoculum rate had little effect on disease incidence in this experiment, suggesting that the presence of inoculum adjacent to plants is more important than its quantity for disease establishment.

The finding that at least one of the cultivars tested has a high degree of resistance in field trials suggests that breeding for disease resistance in New Zealand will be feasible. Conversely, 'Phoenix' had relatively high disease incidence in all trials, and was severely affected in some. While positive selection for disease resistance in wheat breeding programmes is feasible, selection against the most susceptible lines would reduce the incidence of disease in crops.

The variability in ranking of some cultivars between field trials in the present study, as well as in investigations in New Zealand (Cromey et al. 2005) and the United Kingdom (Hollins \& Scott 1983; Clulow \& Wale 1984), suggests the possibility of pathogenic variation within $R$. cerealis. Greenhouse experiments using R. cerealis isolates from different locations provided some evidence for an isolate by cultivar interaction. For instance, the relatively high incidence of sharp eyespot on 'Equinox' inoculated with the Lincoln isolate (from 'Equinox') coincides with

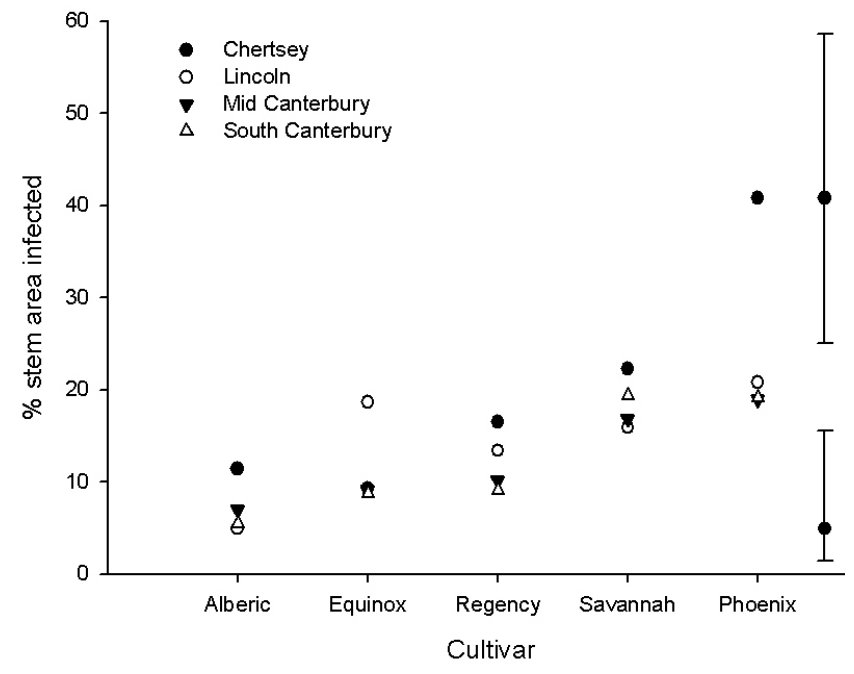

Figure 4 Back-transformed means for each cultivar and 95\% confidence intervals for means of selected points for the percentage stem area of five wheat cultivars inoculated in a greenhouse experiment with four isolates of Rhizoctonia cerealis obtained from different regions in New Zealand. 
that cultivar being the most severely infected in the Lincoln trial in this study and in a previous study (Cromey et al. 2006). Similarly, 'Alberic' was more severely infected with an isolate from 'Alberic' than with other isolates. Further work will be required to determine if these differences are due to genetic variation in the pathogen.

The monitoring of disease progression in commercial wheat crops confirmed the differences between cultivars in resistance to sharp eyespot identified in field trials. The time of sowing had a large effect on development of sharp eyespot in both susceptible and resistant cultivars. As noted previously (Cromey et al. 2006), sharp eyespot incidence and severity was consistently lower in late autumn-sown crops than in earlier sown crops. This result is consistent with overseas studies, where it has been suggested that crop establishment before winter maximises the opportunities for infection before temperatures rise in spring (Polley \& Thomas 1991; Colbach et al. 1997).

Pitt (1964) reported that leaf sheaths on seedlings are more susceptible to infection by $R$. cerealis than those of adult plants and that infection of seedlings was favoured by cool dry conditions. In the present study, lesions tended to be restricted to the outermost leaf sheath of susceptible cultivars during early to mid spring, probably representing primary infection during this highly susceptible growth stage. The lower sharp eyespot incidence in crops sown in March, May and June than crops sown in April suggests that seedling leaf sheaths of April-sown crops were at their most susceptible stages when soil conditions were cool and most conducive to the disease. Pitt (1964) showed that infection at plant maturity results in lesions that are small and generally superficial.

Sharp eyespot incidence (proportion of tillers with visible sharp eyespot lesions on leaf sheaths) declined following the peak in late spring (November). A decrease in visible sharp eyespot on leaf sheaths, resulting from the senescence and loss of outer, infected leaf sheaths, has also been reported in a UK study (Bateman 1993). Recorded disease incidence will drop over time if the rate of spread from outer to inner leaf sheaths is slower than the senescence and loss of these outer leaf sheaths. As in the present study, this decrease was most pronounced during rapid plant development in spring. The progressive, gradual increase in incidence of sharp eyespot lesions extending through inner leaf sheaths is probably the result of secondary spread from primary lesions on outer leaf sheaths. This increase was most prominent in April-sown crops, where primary infection was also greatest.

The similar incidence of sharp eyespot between crops of resistant and susceptible cultivars in early spring suggests that primary infection occurs in both groups of cultivars. The subsequent decline in incidence of visible lesions over following months suggests that secondary spread (leaf sheath to leaf sheath) is limited in the more resistant cultivars, resulting in low severity of disease and negligible disease symptoms during grain fill.

Yield losses from sharp eyespot are usually low where disease incidence is below $10 \%$ (Cromey et al. 2002); therefore, the use of the most resistant cultivars is a useful strategy for avoiding the disease. The resistance of 'Regency' was confirmed in field experiments and in one greenhouse experiment. This cultivar thus provides a basis for a programme of breeding for disease resistance to this disease.

\section{ACKNOWLEDGEMENTS}

This research was funded by the New Zealand Ministry for Science and Innovation (Contract C06X0810) and the Foundation for Arable Research. We thank the operators of the cultivar evaluation trials for trial management and information.

\section{REFERENCES}

Bateman GL 1993. Development of disease symptoms and fungal pathogens on shoot bases in continuous winter wheat, and effects of fungicides. Plant Pathology 42: 595-608.

Clarkson JDS, Cook RJ 1983. Effects of sharp eyespot on yield loss in winter wheat. Plant Pathology 32: 421-428. 
Clulow M, Wale SJ 1984. Resistance of cultivars of wheat, barley and oats to sharp eyespot. Annals of Applied Biology 104 (Suppl. 5): 104-105.

Colbach H, Lucas P, Cavelier N, Carvelier A 1997. Influence of cropping system on sharp eyespot in winter wheat. Crop Protection 16: 415-422.

Cromey MG, Butler RC, Boddington HJ, Moorhead AR 2002. Effects of sharp eyespot on yield of wheat (Triticum aestivum). New Zealand Journal of Crop and Horticultural Science 30: 9-17.

Cromey MG, Butler RC, Munro CA, Shorter SC 2005. Susceptibility of New Zealand wheat cultivars to sharp eyespot. New Zealand Plant Protection 58: 268-272.

Cromey MG, Parkes RA, Fraser PM 2006. Factors associated with stem base and root diseases of New Zealand wheat and barley crops. Australasian Plant Pathology 35: 391-400.

He W, Song Y, Zhang Z 1998. Evaluation of resistance of wheat cultivars to sharp eyespot. Crop Genetic Resources 39: 30-31.

Hollins TW, Scott PR 1983. Resistance of wheat cultivars to sharp eyespot caused by Rhizoctonia cerealis. Annals of Applied Biology 102 (Suppl.): 126-127.

McCullagh P, Nelder JA 1989. Generalized Linear Models. Chapman \& Hall, London, UK.

Pitt D 1964. Studies on sharp eyespot disease of cereals. I. Disease symptoms and pathogenicity of isolates of Rhizoctonia solani Kuhn and the influence of soil factors and temperatures on disease development. Annals of Applied Biology 54: 77-89.

Polley RW, Thomas MR 1991. Surveys of diseases of winter wheat in England and Wales, 19761988. Annals of Applied Biology 119: 1-20.

Schall R 1991. Estimation in generalized linear mixed models with random effects. Biometrika 78: 719-727.

Shi J, Wang Y, Chen H 2000. Screening techniques and evaluation of wheat resistance to sharp eyespot caused by Rhizoctonia cerealis. Acta Phytophylacica Sinica 27: 107-112.

Wiese MV 1987. Compendium of Wheat Diseases. Second edition. APS Press, St Paul, Minnesota, USA.

Zadoks JC, Chang TT, Konzak CF 1974. A decimal code for the growth stages of cereals. Weed Research 14: 415-421. 